\title{
ABUNDANCIA Y REPRODUCCIÓN DEL PELÍCANO PARDO (PELECANUS OCCIDENTALIS) EN DOS PEQUEÑAS BAHÍAS DEL PACÍFICO CENTRAL, MÉXICO
}

\author{
Salvador Hernández-VÁzquez, ${ }^{1}$ Eduardo IñIgo-Elias, ${ }^{2}$ J. Ángel HinoJosa \\ Larios, ${ }^{1}$ Braulio Durand Martínez, ${ }^{3}$ J. A. Rojo-Vázquez ${ }^{1} \&$ Carmen \\ VALADEZ GONZÁLEZ ${ }^{1}$ \\ ${ }^{1}$ Departamento de Estudios para el Desarrollo Sustentable de Zona Costera, Centro Universitario de \\ la Costa Sur, Universidad de Guadalajara. Gómez Farías No. 82, San Patricio-Melaque, Municipio de \\ Cihuatlán, Jalisco 48980, México <sahernan@costera.melaque.udg.mx>. \\ ${ }^{2}$ Cornell Lab of Ornithology, 159 Sapsucker Woods Rd, Ithaca, NY 14850, USA. \\ $<$ eei2@cornell.edu>. \\ ${ }^{3}$ Secretaría de Desarrollo Rural del Gobierno del Estado de Jalisco. Av. Hidalgo \# 1435 Col. \\ Americana, Guadalajara, Jalisco 44100, México. <brauliodurand1@hotmail.com>.
}
Hernández-Vázquez, S., E. Iñigo-Elias, J. Á. Hinojosa Larios, B. Durand Martínez, J. A. Rojo- Vázquez \& C. Valadez González. 2011. Abundancia y reproducción del pelícano pardo (Pelecanus occidentalis) en dos pequeñas bahías del Pacífico central, México. Acta Zoológica Mexicana (n. s.), 27(2): 257-271.

RESUMEN. La abundancia y reproducción del pelícano pardo (Pelecanus occidentalis) se estudió en dos bahías del pacífico central Mexicano, Bahía de Navidad y Bahía Chamela, ubicadas en la costa de Jalisco. De diciembre de 2007 a noviembre de 2009 se registró mensualmente el número de pelícanos pardos en ambas bahías, así como el número de nidos, huevos y pollos en la isla Cocinas. Se midió mensualmente la temperatura superficial del agua, nutrientes $\left(\mathrm{NO}_{2}^{-}, \mathrm{NO}_{3}^{-}, \mathrm{NH}_{4}^{+}\right.$y $\left.\mathrm{PO}_{4}^{-}\right)$y la productividad primaria (clorofila $a$ ). El mayor número de pelícanos se registró de enero a marzo, en ambas bahías. Hubo una mayor abundancia de aves en la Bahía Chamela, principalmente a principios de 2009. El período reproductivo del pelícano pardo se extendió de diciembre a julio en los dos años. El mayor número de nidos se observó en marzo y abril, con un estimado de 480 (90.7 nidos/hectárea) en toda la temporada de anidación del 2008, y de 1,200 (226.8 nidos/hectárea) en 2009. En 2008 se estimaron 1,310 huevos y 800 pollos, mientras que en 2009 se estimaron 3,348 huevos y 2,200 pollos. Tanto el número de aves, como el número de nidos, huevos y pollos registrados en 2009 fueron mayores cuando la temperatura del agua registró sus valores más bajos y la productividad primaria (clorofila $a$ ) y nutrientes, principalmente $\mathrm{NO}_{3}^{-}$y $\mathrm{PO}_{4}^{-}$más altos. Estas variables tienen un efecto directo en la concentración de un mayor número de peces.

Palabras clave: Pelecanus occidentalis, abundancia, reproducción, temperatura, nutrientes, productividad primaria, Jalisco, México. 


\section{Hernández-Vázquez, S., E. Iñigo-Elias, J. Á. Hinojosa Larios, B. Durand Martínez, J. A. Rojo- Vázquez \& C. Valadez González. 2011. Abundance and reproduction of brown pelican (Pelecanus occidentalis) in two bays in the Mexican central Pacific. Acta Zoológica Mexicana (n. s.), 27(2): 257-271.}

ABSTRACT. The abundance and reproduction of the brown pelican (Pelecanus occidentalis) was studied in two bays of the Mexican Central Pacific, Bahía de Navidad and Bahía Chamela, located in the coast of Jalisco, Mexico. The number of brown pelicans in both bays was registered from December 2007 to November 2009, as well as the number of nests, eggs and nestlings in the Cocinas Island. The superficial temperature of the water, the nutrients $\left(\mathrm{NO}_{2}^{-}, \mathrm{NO}_{3}^{-}, \mathrm{NH}_{4}^{+}\right.$y $\left.\mathrm{PO}_{4}^{-}\right)$and the primary productivity (clorofile $a$ ) were measured on a monthly basis. The greater number of pelicans was registered from January to March, in both bays. There were a bigger number of birds in the Chamela bay, mainly in early 2009. The reproductive period of the brown pelican extended from December to July on both years. The largest number of nests was observed in March and April, with an estimate of 480 (90.7 nests/hectares) in the whole nesting season of 2008 and 1,200 (226.8 nests/hectares) in 2009. In 2008 there were an estimate 1,310 eggs and 800 nestlings, while in 2009 there were an estimate 3,348 eggs and 2,200 nestlings. Both, the highest number of birds, as well as the highest number of nests, eggs and nestlings registered in 2009 were present when the temperature of the water registered its lowest values and the primary productivity (clorofile $a$ ) and nutrients, mainly $\mathrm{NO}_{3}^{-}$and $\mathrm{PO}_{4}^{-}$, registered their highest levels. These variables have a direct effect in the concentration of a larger number of fish.

Key Words: Pelecanus occidentalis, abundance, reproduction, temperature, nutrients, primary productivity, Jalisco, Mexico.

\section{INTRODUCCIÓN}

El pelícano pardo (Pelecanus occidentalis), es un ave de gran relevancia ecológica por su distribución geográfica, su diversidad y su biomasa (Schreiber \& Burger 2002). Se encuentra distribuido en las costas del Pacífico, Atlántico e islas del Caribe. En el Pacífico, es el ave marina con mayor distribución, se encuentra desde Columbia Británica hasta Chile e islas Galápagos (Gress \& Anderson 1983; Shields 2002; U.S. FWS 2009). La más reciente estimación de su población en el pacífico Mexicano es de 70,680 parejas anidando, lo cual representa aproximadamente poco más de 141,000 individuos reproductivos (Anderson et al. 2007). Durante la estación reproductiva un mayor número de pelícanos se concentran en las islas del Golfo de California con un estimado de 43,350 parejas (61\%), seguido por la parte sur de California con 11,695 (16.6\%) y el resto se encuentra distribuido en ambientes estuarinos e islas continentales de la parte occidental de México (Anderson et al. 2007).

$\mathrm{Al}$ igual que todas las aves marinas, la distribución y abundancia del pelícano pardo son influenciadas por diferentes variables oceanográficas, pero principalmente por las características fisicoquímicas del agua (Schreiber \& Burger 2002). Por ejemplo, hay gradientes físicos, corrientes, remolinos, surgencias y masas de agua que elevan la concentración de nutrientes, y con ello aumentan la productividad primaria y de los peces que se alimentan de plancton (Ballance \& Pitman 1999). Esta relación ha permitido determinar la existencia de comunidades de aves caracterizadas por las 
diferentes propiedades de las masas de agua donde habitan, debido a que tienen un efecto directo en la distribución y abundancia del alimento (Anderson et al. 1982).

A finales de los 60 's y principio de los 70's el pelícano pardo fue motivo de diversas investigaciones como consecuencia del descenso de su población causado por contaminantes (Schreiber 1980; Wickliffe \& Bickham 1998); debido al declive poblacional, el Servicio de Pesca y Vida Silvestre (FWS por sus siglas en inglés) la incluyó en la Ley de Especies en Peligro de Extinción (ESA por sus siglas en inglés) en 1973 (U.S. FWS 2009). A partir de la prohibición del uso de DDT en EUA la población de pelícanos se ha ido recuperando, al grado de que en 2009 se eliminó de la lista en la Ley de Especies en Peligro de Extinción. Esta acción se basó en una revisión y monitoreo de su población de varios años, que indican que la especie ya no está en peligro de extinción, o susceptible de serlo en un futuro previsible. Sin embargo, seguirá siendo protegida por las disposiciones del Tratado de Aves Migratorias (U.S. FWS 2009).

Recientemente se han publicado diversos estudios del pelícano pardo, algunos se han enfocado a parásitos (Dronen et al. 2003; Zamparo et al. 2005; Reeves et al. 2006; Mattiucci et al. 2008), contaminantes (Wickliffe \& Bickham 1998), monitoreo poblacional (Godínez-Reyes et al. 2006) y estimaciones de sus poblaciones (Anderson et al. 2007). Estos estudios han sido realizados al norte de México, particularmente en California y el Golfo de California donde se concentra el mayor número de aves (Grees \& Anderson 1983; Shields 2002; Anderson et al. 2007).

En el Pacífico central Mexicano y particularmente en la costa de Jalisco se han realizado pocos estudios, siendo escasos los relativos a la abundancia y reproducción. Por ejemplo, Anderson et al. (2007) reporta 70 nidos en las islas Los Arcos, Bahía Banderas, y solo hace mención que las islas de Bahía Chamela son adecuadas para anidar, pero sin dar mayor información sobre ello. Los pocos estudios realizados en la costa de Jalisco han sido enfocados de forma general a las aves acuáticas en humedales, y sólo presentan datos de abundancias mensuales o anuales del pelícano pardo en algunos humedales (Hernández-Vázquez 2000; Hernández-Vázquez \& Mellink 2001; Hernández-Vázquez et al. 2002; Hernández-Vázquez 2005). En las áreas insulares sólo se cuenta con los trabajos realizados en las islas Marietas, Nayarit (Gaviño \& Uribe 1981; Rebón-Gallardo 2000). En los trabajos publicados por Hernández-Vázquez (2000), Hernández-Vázquez \& Mellink (2001) abordan las aves marinas en general pero dan información limitada del pelícano pardo. Posteriormente, Hernández-Vázquez et al. (2010) publican información generada en el año 2000 en la que abordan algunos aspectos de la biología reproductiva del pelícano pardo en la isla Cocinas, en la Bahía Chamela. Considerando lo anterior, el objetivo de este estudio fue generar información durante dos años (2008 y 2009) sobre la abundancia y anidación del pelícano pardo y su relación con algunas variables oceanográficas en dos bahías de la costa de Jalisco, Bahía de Navidad y Bahía Chamela. Esta informa- 
ción además de contribuir al escaso conocimiento de la especie en el pacífico central Mexicano, servirá como punto de comparación para estudios futuros en la zona y otras áreas de distribución del pelícano pardo, de tal forma que pueda ser usada para plantear medidas de mitigación y control que ayuden a la conservación de la especie y sus hábitats, evitando así que nuevamente pueda estar amenazada o que presente declives poblacionales que indiquen problemas en la región.

\section{ÁREA DE ESTUDIO}

El estudio se realizó en la Bahía de Navidad y Bahía Chamela. La Bahía de Navidad se localiza en el extremo sur de la costa del estado de Jalisco (19¹0'30" N, $104^{\circ} 42^{\prime} 45^{\prime}$ ' O y $\left.19^{\circ} 12^{\prime} 50^{\prime \prime} \mathrm{N}, 104^{\circ} 41^{\prime} 30^{\prime \prime} \mathrm{O}\right)$. Cuenta con zonas de fondos rocosos

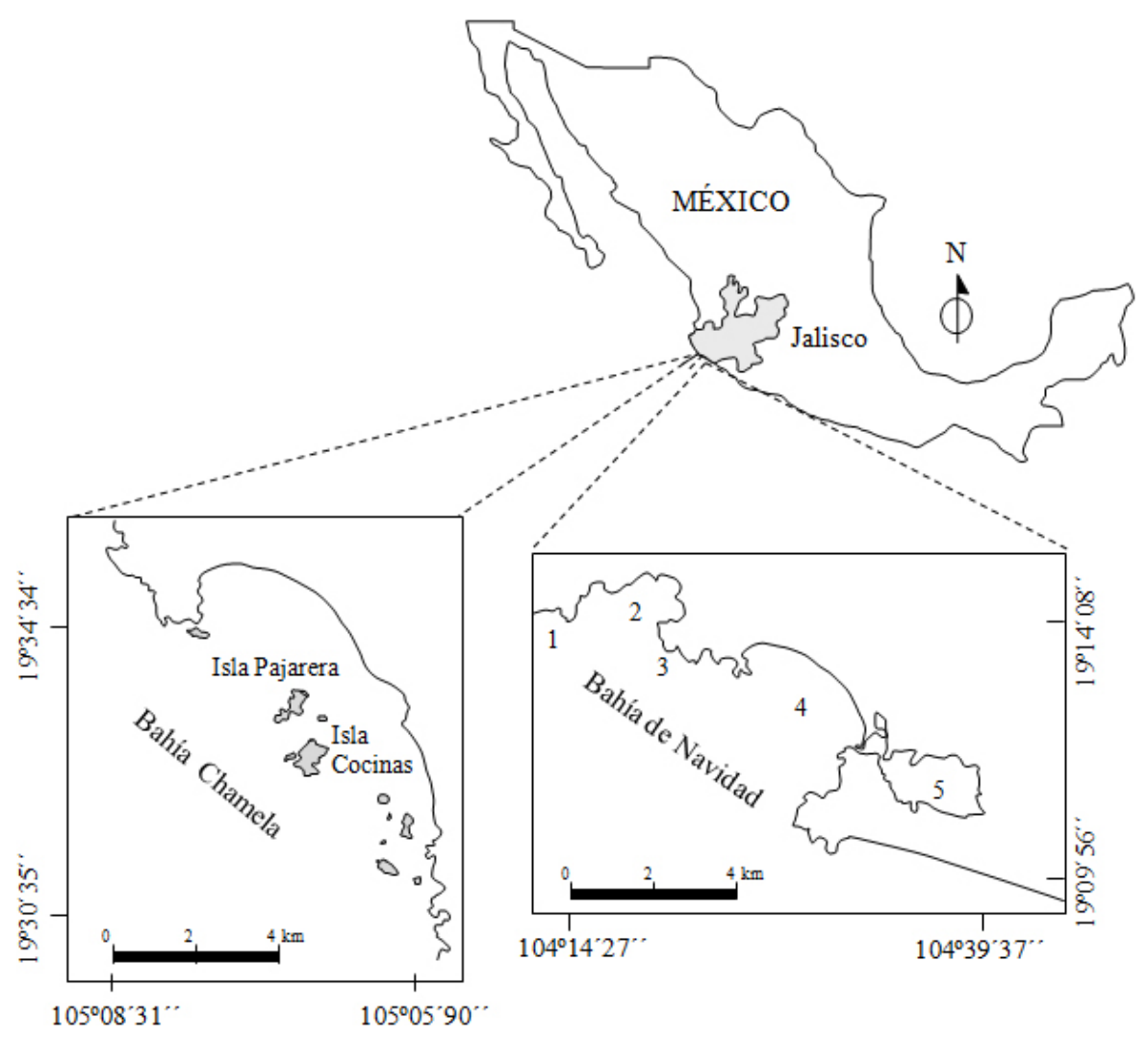

Figura 1. Ubicación geográfica de la Bahía de Navidad y Bahía Chamela, Jalisco, México. 1 = Punta Corrales, 3 = Caleta Cuastecomatito, $2=$ Bahía de Cuastecomates, $4=$ Bahía de Melaque y $5=$ Laguna Barra de Navidad. 
(Punta Corrales y Caleta Cuastecomatito), fondos blandos (Bahía de Cuastecomates y Bahía de Melaque) y fondos blandos con piedras pequeñas. Esta bahía tiene una gran actividad urbana y turística debido a los poblados de Melaque y Barra de Navidad (Fig. 1), además de la laguna Barra de Navidad que tiene un intenso tráfico de embarcaciones pequeñas de pescadores y yates.

La Bahía Chamela se encuentra a $61 \mathrm{~km}$ al noroeste de la Bahía de Navidad. Se ubica en el municipio de la Huerta, en la parte central de la costa de Jalisco (19 $35^{\prime}$ '19" $\mathrm{N}, 105^{\circ} 08^{\prime} 37^{\prime \prime} \mathrm{O}$ y $19^{\circ} 30^{\prime} 14^{\prime \prime} \mathrm{N}, 105^{\circ} 04^{\prime} 41^{\prime \prime} \mathrm{O}$ ), entre las desembocaduras de los ríos Cuitzmala y San Nicolás. Se extiende desde Punta Rivas en el extremo norte de la bahía hasta punta Etiopía al sur, a lo largo de $11.5 \mathrm{~km}$ en dirección noroeste-sureste. En la bahía hay 13 pequeñas islas e islotes, de las cuales las más grandes son isla Cocinas e isla Pajarera. La vegetación predominante de las islas es bosque tropical caducifolio, matorral xerófilo de acantilados y vegetación de dunas costeras (Rzedowski 1978). La Bahía Chamela ha sido incorporada al Sistema Nacional de Áreas Naturales Protegidas, y las 13 pequeñas islas que se encuentran en ella han sido decretadas para México bajo la categoría de Santuario en el Diario Oficial de la Federación el 13 de Junio de 2002.

Cocinas es la isla más grande de la bahía Chamela con una superficie de 32 hectáreas $\left(19^{\circ} 32^{\prime} 49^{\prime \prime} \mathrm{N}, 105^{\circ} 06^{\prime} 35^{\prime \prime} \mathrm{O}\right)$ y se encuentra ubicada a $1,900 \mathrm{~m}$ de la costa. La vegetación predominante en la parte oriente está formada principalmente por cuachalalate (Amphipterygium sp.) y en menor proporción por órganos (Pachycereus pecten-aboriginum) que alcanzan hasta $8-10 \mathrm{~m}$ de altura. Durante el mes de octubre, es característica la presencia de la yerba (Elytaria sp.) que forma un manto en el piso. En la cima y en la ladera poniente, las especies están achaparradas. También se encuentran arbustos de Bursera excelsa var. favonialis, Manihot colimensis y Capparis indica (Sánchez-Hernández \& Pérez-Jiménez 1972).

\section{MÉTODO}

De diciembre de 2007 a noviembre de 2009 (24 meses), se realizaron visitas mensuales a la Bahía de Navidad y Bahía Chamela, con el fin de monitorear la población del pelícano pardo. Se realizaron recorridos por las mañanas $(07: 00 \mathrm{~h}$ a 10:00 h) a lo largo de las dos bahías. Se usó una lancha de siete metros de eslora, con un motor fuera de borda de 80 caballos de fuerza, a una velocidad promedio de $20 \mathrm{~km} / \mathrm{hora}$. En el caso de la Bahía Chamela, la información obtenida en cada recorrido se complementó con observaciones realizadas en las playas e islas.

La colonia reproductiva del pelícano pardo se monitoreó sólo en la isla Cocinas por ser el sitio de mayor concentración de nidos en la Bahía Chamela y en todo Jalisco (Hernández-Vázquez et al. 2010). Con base a observaciones en años anteriores el mayor número de nidos se concentra en la parte norte de la isla, en un área aproxi- 
mada de 52,900 $\mathrm{m}^{2}$. Con el fin de disminuir las perturbaciones causadas por nuestra presencia, dentro de esta área se estableció un cuadrado de $65 \times 40 \mathrm{~m}$ para realizar los muestreos. Los nidos registrados en el cuadrado se marcaron con una cinta plástica visible y un número consecutivo de acuerdo a la fecha de su aparición, y mensualmente se contaron los nidos, huevos y pollos que aparecían dentro del cuadrado. La información generada en el cuadrado se extrapoló en todo el polígono de anidación $\left(52,900 \mathrm{~m}^{2}\right)$, dado que el cuadrado era representativo de la densidad que pueden tener los nidos en el total de la isla. Este método de extrapolación permite estimar la abundancia de aves en un área determinada cuando no se puede monitorear o contar toda la población (Howes \& Bakewell 1989). Los nidos se contaron por dos personas durante la mañana, entre las 07:00 h y 10:00 h, con el fin de evitar que las aves abandonaran los nidos y que los huevos y pollos quedaran expuestos a la hora de mayor calor.

Debido a que los pelícanos se observaron alimentándose en grandes cantidades, se realizaron colectas de peces con el fin de capturar las posibles presas de las que se alimentaban. Estas colectas se realizaron sólo en enero y febrero en ambas bahías y en los sitios donde las aves se alimentaban. Las especies colectadas se compararon con algunos regurgitados recolectados en los sitios de anidación. Para la captura de los peces se usó una tarraya.

La temperatura superficial del agua, nutrientes (nitritos $\mathrm{NO}_{2}^{-}$, nitratos $\mathrm{NO}_{3}^{-}$, amonio $\mathrm{NH}_{4}^{+} \mathrm{y}$ ortofosfato $\mathrm{PO}_{4}^{-}$) y clorofila $a$ se midieron para determinar los meses de mayor productividad del agua en las dos bahías. Se hicieron pruebas de correlación de estas variables con la abundancia de aves y de nidos para tratar de determinar la existencia de alguna relación. Asimismo, mediante la medición de dichas variables se puede determinar de forma indirecta el período de mayor disponibilidad de peces (Ballance \& Pitman 1999; Shealer 2002). En cada bahía se seleccionaron seis puntos a lo largo de una línea perpendicular a la costa (un kilómetro separado un punto de otro). En cada punto se midió mensualmente la temperatura superficial del agua y se colectaron muestras de agua para su posterior análisis en el laboratorio y la determinación de nutrientes y clorofila $a$. La temperatura se tomó con un multiparámetros digital YSI (modelo $556 \mathrm{MPS}$ ). Los $\mathrm{NO}_{2}^{-}$y NO colorimétrico, el $\mathrm{NH}_{4}^{+}$con el método colorimétrico de azul indofenol, los $\mathrm{PO}_{4}^{-}$con el método colorimétrico de azul de molibdeno. La clorofila $a$ se calculó por el método de extracción por acetonas. Estos métodos están descritos en el Manual de Prácticas de Agua de Mar (Parsons et al. 1984).

Se usó la prueba de Kolmogorov-Smirnov para probar si los datos presentaban una distribución normal. Debido a que estos no eran normales $(\mathrm{K}-\mathrm{S}, p<0.05)$ se usó la prueba de chi-cuadrada $\left(\chi^{2}\right)$ con el fin de determinar las diferencias significativas en el número de pelícanos entre años en una misma bahía y entre bahías. La diferencia de las proporciones en el número de huevos/nido entre 2008 y 2009 se realizó con una chi-cuadrada. En todas las pruebas se usó un $\alpha=0.05$. La relación entre la abun- 
dancia de aves y la temperatura, nutrientes y clorofila $a$ se determinó con una prueba de correlación de rango de Spearman (Zar 1974).

\section{RESULTADOS}

Número de pelícanos y abundancia temporal. En Bahía de Navidad el número de pelícanos registrado mensualmente presentó diferencias significativas entre años $\left(\chi^{2}\right.$ $=2843.4, \mathrm{gl}=23, p<0.05)$. La mayor abundancia de aves se observó en enero del 2008 y enero del 2009 con 280 y 3500 individuos, respectivamente (Fig. 2a). En la Bahía Chamela el número de aves estimado mensualmente fue menor en 2008 que

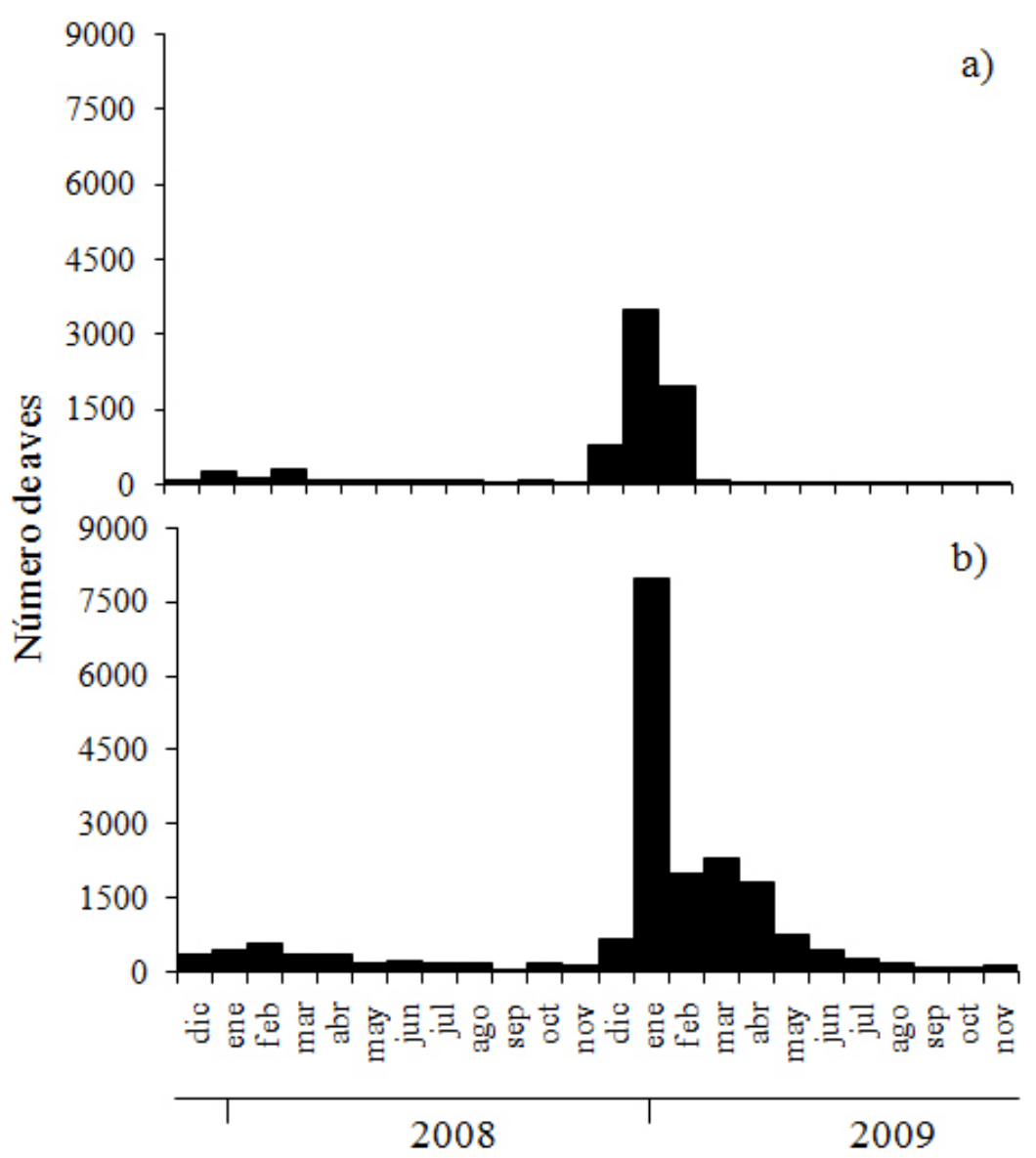

Figura 2. Número mensual de aves de pelícano pardo observadas en la Bahía de Navidad (a) y Bahía Chamela (b), durante 2008 y 2009. 
en $2009\left(\chi^{2}=2615.9, \mathrm{gl}=23, p<0.05\right)$. En 2008 se registraron más aves de enero a abril, con su máxima abundancia en febrero (580 aves), mientras que en 2009 se registraron más aves en enero a abril, siendo enero el mes cuando se observó el mayor número de aves (8000 aves) (Fig. 2b). De las 8000 aves observadas en la Bahía Chamela, la mayoría se encontró en la playa, en el área donde se comunica el estero Chamela con el mar (5000 aves), en las áreas rocosas de la isla San Agustín (2000) e isla San Pedro (1000), islas situadas a $2.3 \mathrm{~km}$ al sureste de la isla Cocinas.

Aunque el patrón en las abundancias mensuales en el número de aves es similar en ambos años, este es mucho mayor en 2009. La comparación del número de pelícanos observados en los dos años también presentó diferencias significativas entre las dos bahías, siendo mayor en la Bahía Chamela $\left(\chi^{2}=3239.8, \mathrm{gl}=47, p<0.05\right)$.

Distribución espacial de nidos y cronología reproductiva. Se registraron nidos en varias partes de la isla Cocinas, pero su mayor concentración se observó en la parte norte. Los nidos se construyeron en arbustos y árboles, a una altura entre 1.5 y $6 \mathrm{~m}$ del suelo.

El período reproductivo del pelícano pardo se extendió de diciembre a julio en ambos años. En 2008 hubo un total de 480 nidos, con una densidad de 90.7 nidos/ hectárea, mientras que en 2009 se estimaron 1200 nidos y una densidad de 226.8 nidos/hectárea. En 2008 el mayor número de nidos se registró en marzo y abril, con 238 y 290 nidos, respectivamente. En el 2009 se registró un patrón similar, observando el mayor número en marzo y abril, con 734 y 660 nidos, respectivamente (Fig. 3). El número de huevos por nido fue de dos a cuatro; de 50 nidos observados en 2008, el $30 \%$ tuvo dos huevos, el 67\% tres huevos y el 3\% cuatro huevos. En 2009 fueron muy similares las proporciones, ya que de 50 nidos el $25 \%$ tuvo dos huevos, $71 \%$ tres hue-

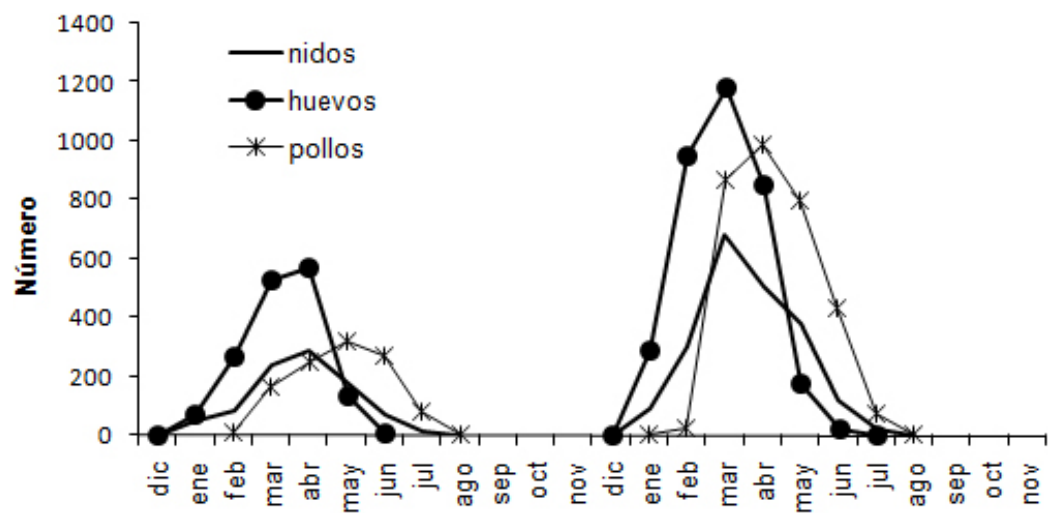

Figura 3. Número mensual de nidos, huevos y pollos del pelícano pardo registrados en la isla Cocinas, durante la temporada de anidación 2008 y 2009. 
vos y el $4 \%$ cuatro huevos $\left(\chi^{2}=0.713, \mathrm{gl}=5, p>0.05\right)$. En 2008 se estimaron 1334 huevos en todo el período de anidación, con sus mayores abundancias en marzo (526) y abril (570). En 2009 se estimaron un total de 3348 huevos, siendo febrero, marzo y abril cuando se observó el mayor número de huevos. El número de pollos también presentó diferencias entre años; en 2008 se estimaron 800 pollos en todo el período reproductivo, siendo mayo el mes cuando se registró el mayor número, mientras que 2009 hubo 2200 pollos, con su mayor abundancia en abril (Fig. 3).

Variables oceanográficas. La temperatura superficial del agua presentó una clara estacionalidad a lo largo del período de estudio. En Bahía de Navidad y Bahía Chamela las temperaturas más bajas se registraron de diciembre a junio del 2008 y 2009, mientras que los valores más altos se observaron de julio a noviembre en ambos años (Fig. 4a y 4b). Los nutrientes presentaron fluctuaciones en las dos bahías. Los $\mathrm{NO}_{3}^{-}$y $\mathrm{PO}_{4}^{-}$son los nutrientes que registraron valores más altos, principalmente cuando la

a). Bahía de Navidad
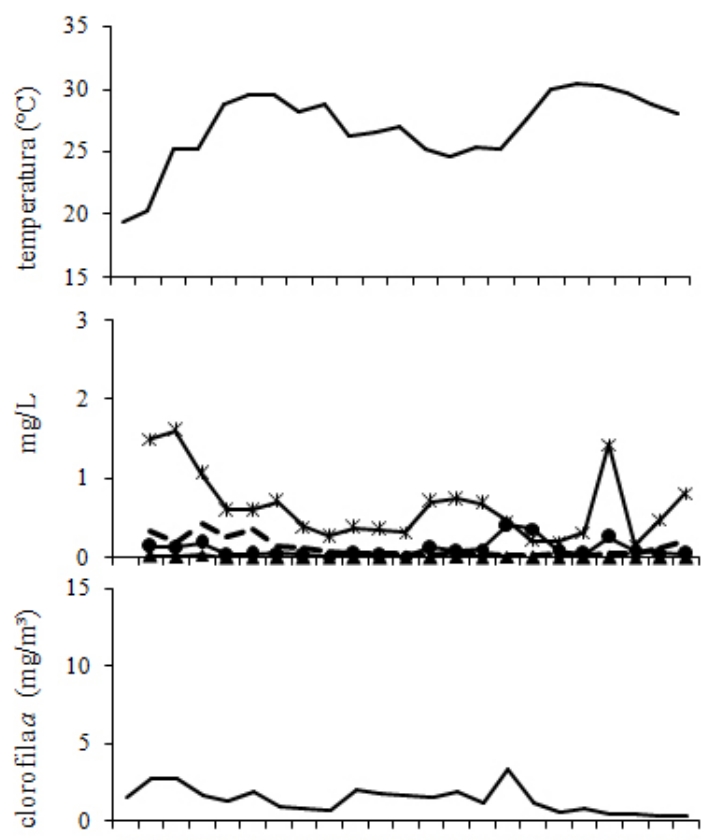

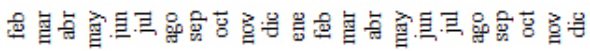

b). Bahía Chamela
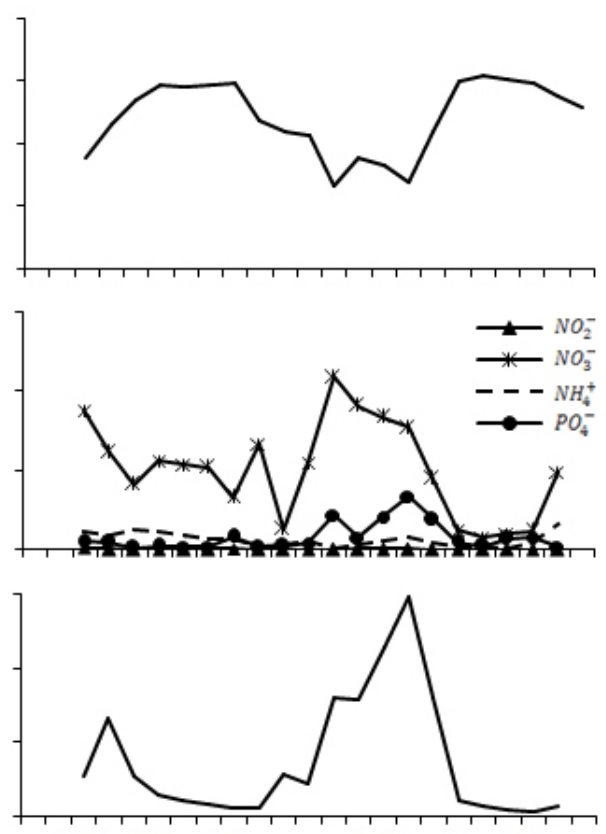

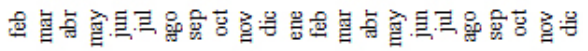
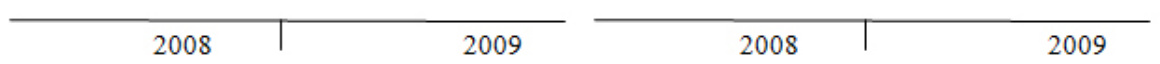

Figura 4. Temperatura superficial del agua, clorofila $a$ y nutrientes registrados mensualmente durante 2008 y 2009 en Bahía de Navidad (a) y Bahía Chamela (b). 
temperatura del agua es más fría. Este patrón es más claro en la Bahía Chamela. La clorofila $a$ registró una variación entre bahías. En la Bahía de Navidad se mantuvo constante en los dos años de estudio, con ligeros incrementos en marzo $\left(2.69 \mathrm{mg} / \mathrm{m}^{3}\right)$ y abril $\left(2.74 \mathrm{mg} / \mathrm{m}^{3}\right)$ del 2008 , y mayo $\left(3.33 \mathrm{mg} / \mathrm{m}^{3}\right)$ del 2009. En la Bahía Chamela la clorofila $a$ registró valores más altos que en Bahía de Navidad. En esta bahía se observaron dos incrementos; uno en mayo del $2008\left(6.7 \mathrm{mg} / \mathrm{m}^{3}\right)$ y otro de febrero a junio, siendo mayo el mes cuando se registró el valor más alto $\left(14.8 \mathrm{mg} / \mathrm{m}^{3}\right)$. Estos incrementos en la clorofila $a$ se presentaron cuando la temperatura del agua es más fría y los niveles de nutrientes más altos (Fig. 4a y 4b).

Al relacionar estas variables con la abundancia mensual de los pelícanos, se encontró que en Bahía de Navidad no hubo una correlación significativa entre el número de aves y la temperatura $(\mathrm{r}=-0.39, p>0.05)$, nutrientes $\left(\mathrm{NO}_{2}^{-}, \mathrm{r}=0.25, p>0.05\right.$; $\mathrm{NO}_{3}^{-}, \mathrm{r}=0.30, p>0.05 ; \mathrm{NH}_{4}^{+}, \mathrm{r}=0.32, p>0.05$ y $\left.\mathrm{PO}_{4}^{-}, \mathrm{r}=-0.25, p>0.05\right)$ y clorofila $a(\mathrm{r}=0.32, p>0.05)$. Sin embargo, en la Bahía Chamela sí se observó una correlación significativa con estas variables. La mayor abundancia de pelícanos se registró con la temperatura más baja $(\mathrm{r}=-0.79, p<0.05)$ y los valores más altos de nutrientes, principalmente $\mathrm{NO}_{3}^{-}(\mathrm{r}=0.54, p<0.05)$ y $\mathrm{PO}_{4}^{-},(\mathrm{r}=0.50, p<0.05)$ y clorofila $a(\mathrm{r}=$ $0.82, p<0.05)$. El número de nidos registrados en la isla Cocinas también registró una correlación significativa con la temperatura $(\mathrm{r}=-0.78, p<0.05), \mathrm{NO}_{3}^{-}(\mathrm{r}=0.73$, $p<0.05), \mathrm{PO}_{4}^{-},(\mathrm{r}=0.59, p<0.05)$ y clorofila $a(\mathrm{r}=0.86, p<0.05)$.

\section{DISCUSIÓN}

El número de pelícanos pardos registrado en Bahía de Navidad fue menor al observado en Bahía Chamela durante el estudio. Estas diferencias pueden atribuirse a las características de cada bahía. En la Bahía Chamela se encuentran 13 islas e islotes que proporcionan hábitats adecuados para descansar y anidar, mientras que Bahía de Navidad carece de islas y las aves han sido observadas alimentándose sólo en la mañana y tarde; después de esto se desplazan a otros sitios fuera de la bahía. Otro aspecto importante a considerar es la presencia de asentamientos humanos y desarrollos turísticos en la Bahía de Navidad. Ya se ha documentado en otros sitios que las actividades derivadas de estos asentamientos tienen impactos negativos en la abundancia de las aves (Rodgers \& Schwikert 2002).

El número de pelícanos pardos observado en ambas bahías, así como el número de nidos registrados en la isla Cocinas es mucho menor al reportado en varias islas aledañas a la península de Baja California, como Las Ánimas (3800 nidos), San Lorenzo (6000 nidos), Ángel de la Guarda (2300 nidos) y San Luis (4800 nidos) (Godínez-Reyes et al. 2006). En las islas Marietas, Nayarit se reportan menos de 2000 individuos (Gaviño \& Uribe 1981; Rebón-Gallardo 2000). Aunque el número de aves observadas en la Bahía Chamela y Bahía de Navidad es relativamente bajo 
comparado con las islas de Baja California, el elevado número de aves observado en 2009 no había sido registrado en años anteriores en la costa de Jalisco. Por ejemplo, algunos estudios previos enfocados a las aves acuáticas reportaron anualmente entre 1000 y 2000 pelícanos en los humedales de la costa de Jalisco (Hernández-Vázquez 2000; Hernández-Vázquez \& Mellink 2001; Hernández-Vázquez et al. 2002). Sin embargo, considerando la revisión de las poblaciones de pelícanos pardo que hace Anderson et al. (2007), podemos considerar que la Bahía de Chamela concentró en 2009 la población más numerosa de pelícanos del pacífico central Mexicano, desde el sur de Sinaloa hasta Guerrero.

El aumento exponencial de pelícanos entre enero y febrero de 2009 fue observado en otras zonas de México. Con base a observaciones de campo, en enero de 2009 se registraron desplazamientos continuos de pelícanos por la costa del pacífico Mexicano entre Manzanillo, Colima (Hernández-Vázquez, observación personal) y la Bahía de Ixtapa Zihuatanejo, Guerrero (C. Madrazo y R. Hernández, com. pers.). Este incremento de pelícanos a principios de año es un patrón que se presenta año con año en la costa sur de Jalisco debido a que un mayor número de pelícanos se concentran para anidar, principalmente en la Bahía Chamela. Sin embargo, en 2009 el número de pelícanos fue mayor al registrado en el 2000 (Hernández-Vázquez et al. 2010) y 2008 en esta misma bahía. Se desconoce la causa de estas diferencias, pero hay varios factores que pudieron contribuir; por ejemplo, disturbios humanos (Anderson 1988), enfermedades (Work et al. 1993), parásitos (Dronen et al. 2003), contaminantes (Wickliffe \& Bickham 1998) o variaciones en las condiciones oceanográficas (Schreiber \& Burger 2002). En el sur de California estas fluctuaciones han estado asociadas con variaciones en la distribución y abundancia del alimento asociado con eventos de El Niño Oscilación del Sur (Anderson et al.1982; Shields 2002; Anderson et al. 2007). En el presente estudio es probable que las diferencias interanuales estén asociadas con los cambios en las condiciones oceanográficas, debido a que en 2009 la temperatura del agua de mar es más baja y la productividad primaria más alta que en 2008. Estas condiciones contribuyen al incremento y disponibilidad del alimento de los pelícanos (Anderson et al. 1982, 2007; Ballance \& Pitman 1999; Schreiber \& Burger 2002).

Aunque la dieta de los pelícanos no es el tema central de este trabajo, en enero y febrero del 2009 se realizaron colectas de peces en las dos bahías. Entre las especies de peces más abundantes colectadas estuvieron el ojotón (Selar crumenophthalmus), la sardina crinuda o machete de hebra (Opisthonema libertate), la sardina machete (Pliosteostoma lutipinnis) y las anchovetas (Anchovia macrolepidota, Anchoa lucida). Estas especies de peces se colectaron en los sitios de mayor concentración de pelícanos y en los momentos en que se alimentaban. Gress \& Anderson (1983) mencionan que los pelícanos pueden desplazarse de 30 a $50 \mathrm{~km}$ de sus colonias de anidación en busca de alimento. En nuestro estudio se observaron alimentándose diariamente en la 
mañana dentro de las bahías, por lo que es altamente probable que lo hicieran de estas especies de peces. Analizamos cuatro regurgitados colectados de los nidos en la isla Cocinas y contenían anchovetas (A. macrolepidota y A. luvida). Aunque el número de regurgitados es bajo, puede mostrarnos que esas especies forman parte de su dieta.

A partir de mayo-junio cuando la temperatura del agua empezó a incrementarse y la clorofila $a$ y nutrientes empezaron a descender, se observó también un descenso en el número de pelícanos. Probablemente ello represente una respuesta a una disminución de sardinas y anchovetas (Aguilar-Palomino \& Kosonoy, com. pers.), aparentemente causado por la disminución de la productividad primaria en el mar.

Como ya ha sido mencionado anteriormente, el incremento en el número de pelícanos en la Bahía de Navidad y Bahía Chamela se asoció al inicio del período reproductivo, que se extendió de diciembre a julio, con su mayor abundancia de nidos en marzo, abril y mayo. Este patrón en la cronología de puesta es similar al registrado en 2000 en la isla Cocinas, en la Bahía Chamela (Hernández-Vázquez et al. 2010), aunque hubo diferencias en el número de nidos. Es probable que estas variaciones interanuales estén relacionadas con la abundancia o ausencia del alimento (Anderson et al. 1980, 1982, 2007; Anderson \& Gress 1983).

La mayor abundancia de pelícanos en Bahía de Navidad y Bahía Chamela se registró a principios de cada año, de enero a abril. En un estudio realizado en el centro y norte de California, se encontró que la mayor abundancia de pelícanos se presenta de agosto a noviembre, para posteriormente desplazarse al sur de California cuando las variables oceanográficas cambian y hay una mayor abundancia de alimento, como la anchoveta (Briggs et al. 1981, 1983). Tanto el mayor número de pelícanos como el mayor número de nidos se registró en invierno en nuestra área de estudio, cuando la temperatura superficial del agua es más fría y los valores más altos de nutrientes y clorofila $a$. Durante este período, la costa de Jalisco se encuentra bajo la influencia de la advección de la Corriente de California, así como de la corriente superficial que sale del Golfo de California (Filonov et al. 2000). Este sistema de corrientes incrementa la productividad primaria en la zona de estudio, misma que favorece una mayor concentración de peces que se alimentan de plancton, como la anchoveta (Mann \& Lazier 1991; Ayón et al. 2008). Este aumento en la disponibilidad de alimento favorece el incremento en el número nidos de pelícanos (Anderson et al. 1980, 1982; Anderson \& Gress 1983).

Adicionalmente, es importante mencionar que junto con el aumento en el número de aves observadas a principios del 2009 en Bahía de Navidad, se registraron 80 individuos muertos entre enero y marzo de 2009, así como 120 en Bahía Chamela. En otros sitios, como en la costa de California también se reportó la muerte de 460 pelícanos entre diciembre de 2008 y enero de 2009 (Miller 2009). En California como en otros sitios del pacífico Mexicano, incluyendo Jalisco, no ha quedado clara la causa de muerte de estas aves. Sin embargo, hay registros en California de la muerte 
masiva de pelícanos causada por la ingesta de anchovetas y cangrejos que contenían en sus vísceras altas concentraciones de ácido domoico (Work et al. 1993; Hallegraeff et al. 1995), que es una sustancia toxica producida por diatomeas del género Pseudonitzschia spp. (Work et al. 1993). Aunque en Jalisco no se realizó la necropsia en las aves muertas, sí se registraron mareas rojas en los meses cuando se observaron los pelícanos muertos, siendo Pseudonitzschia spp. una de las especies de algas más abundantes registradas (Franco-Gordo, datos no publicados). Es probable por ello, que eventos de mareas rojas con la diatomea que produce ácido domoico hayan sido causantes de la mortalidad de pelícanos por intoxicación en nuestra área de estudio.

Los resultados obtenidos en 2008 y 2009 muestran que tanto el mayor número de pelícanos registrados en la Bahía de Navidad y Bahía Chamela, así como el mayor número de nidos observados en esta última bahía se presentaron a principios de cada año. Sólo en Bahía Chamela hubo una correlación con las variables oceanográficas medidas, encontrando que el mayor número de aves y nidos se observaron cuando la temperatura del agua es más baja y los nutrientes y clorofila $a$ son más altos. Aparentemente estas condiciones oceanográficas permiten que se concentre un mayor número de peces que se alimentan de plancton, como la sardina y anchoveta, mismas que como ya ha sido documentado, forman parte importante en la dieta del pelícano pardo (Anderson et al. 1980, 2007). El número de pelícanos y nidos registrados en la Bahía Chamela representa la colonia más numerosa reportada en las islas del pacífico central Mexicano, desde Nayarit hasta Guerrero. Es importante por tanto monitorear la zona para mantener el número de pelícanos estable.

AGRADECIMIENTOS. Se agradece a Julio Cesar López, Sergio Serrano, Armando Martínez, Enrique Woodman, José Mitoma y Raúl Enrique Lara por el apoyo en el trabajo de campo. Al Departamento de Estudios para el Desarrollo Sustentable de Zona Costera, Universidad de Guadalajara por el apoyo logístico. A Ricardo Rodríguez Estrella y dos revisores anónimos por sus valiosos comentarios que ayudaron a mejorar el escrito. Este trabajo ha sido financiado por el Centro Universitario de la Costa Sur, Universidad de Guadalajara a través de sus programas de Investigación-2008 y por el Programa de Mejoramiento del Profesorado (PROMEP: 103.5/07/2449).

\section{LITERATURA CITADA}

Anderson, D. W., F. Gress., K. F. Mais \& P. R. Kelly. 1980. Brown Pelicans as anchovy stock indicators and their relationships to commercial fishing. CalCOFI Report, 21: 54-61.

Anderson, D. W., F. Gress \& K. F. Mais. 1982. Brown Pelicans: influence of food supply on reproduction. Oikos, 39: 23-31.

Anderson, D. W. \& F. Gress. 1983. Status of a Northern population of California Brown Pelicans. Condor, 85: 79-88.

Anderson, D. W. 1988. In my experience: Dose-response relationship between human disturbance and Brown Pelican breeding. Wildlife Society Bulletin, 16: 339-345.

Anderson, D. W., C. J. Henny., C. Godínez-Reyes., F. Gress., E. L. Palacios., K. Santos del Prado. \& J. Bredy. 2007. Size of the California Brown Pelican metapopulation during a Non-El Niño year. 
Reston, Virginia, U.S. Geological Survey, Open-File Report 2007-1299.

Ayón, P., G. Swartzman., A. Bertrand., M. Gutierrez. \& S. Bertrand. 2008. Zooplankton and forage fish species off Peru: large-scale bottom-up forcing and local-scale depletion. Progress in Oceanography, 79: 208-214.

Ballance, L. T. \& R. L. Pitman. 1999. Foraging ecology of tropical seabirds, pp. 2057-2071. In: N.J. Adams and R.H. Slotow (Eds.) Proc. 22 Int. Ornithol. Congr., Durban: 2057-2071. Johannesburg: BirdLife South Africa.

Briggs, K. T., D. B. Lewis., W. B. Tyler \& G. L. Hunt. 1981. Brown pelicans in southern California: Habitat use and environmental fluctuations. Condor, 83: 1-15.

Briggs, K. T., W. B. Tyler., D. B. Lewis., P. R. Kelly \& D. A. Croll. 1983. Brown Pelicans in Central and Northern California. Journal of Field Ornithology, 54: 353-373.

Dronen, C. K., C. K. Blend, \& C. K. Anderson. 2003. Endohelminths from the Brown Pelican, Pelecanus occidentalis, and the American White Pelican, Pelecanus erythrorhynchus, from Galveston Bay, Texas, U.S.A., and a checklist of pelican parasites. Comparative Parasitology, 70: 140-154.

Filonov, A. E., I. E. Tereshchenko., C. O. Monzón., M. E. González-Ruelas \& E. Godínez-Domínguez. 2000. Seasonal variability of the temperature and salinity field in the coast zone of the states of Jalisco and Colima, México. Ciencias Marinas, 26: 303-321.

Gaviño, G. \& Z. Uribe. 1981. Distribución, población y época reproductiva de las aves de las islas Tres Marías, Jalisco. Anales del Instituto de Biología, Serie Zoología, 51: 505-524.

Godínez-Reyes, C., K. S. del Prado-Gasca., H. Zepeda-López., A. Aguirre., D. W. Anderson., A. P. González., E. Velarde \& A. Zavala-González. 2006. Monitoreo de poblaciones y condición de salud de aves marinas y lobos marinos en islas del norte del Golfo de California, México. Gaceta Ecológica, 81: 31-45.

Gress, F. \& D. W. Anderson. 1983. California Brown Pelican recovery plan. Prepared for the Fish and Wildlife Service Endangered Species Office, U.S. Fish and Wildlife Service, Portland, OR.

Hallegraeff, G. M., D. M. Anderson \& A. D. Cembella. 1995. Manual of Harmful Marine Microalgae. Intergovernmental Oceanographic Commission (UNESCO). $551 \mathrm{p}$

Hernández-Vázquez, S. 2000. Aves acuáticas del estero La Manzanilla, Jalisco, México. Acta Zoológica Mexicana, nueva serie, 80: 143-153.

Hernández-Vázquez, S. \& E. Mellink. 2001. Coastal waterbirds of El Chorro and Majahuas, México, during the non-breeding season, 1995-1996. Revista Biología Tropical, 49: 357-365.

Hernández-Vázquez, S., H. De la Cueva S. \& J. A. Rojo-Vázquez. 2002. Análisis comparativo de la avifauna del estero Majahuas (Jalisco, México) entre un evento El Niño y un año no Niño. Boletín del Centro de Investigaciones Biológicas, 36: 94-112.

Hernández-Vázquez, S. 2005. Aves acuáticas de la laguna de Agua Dulce y esteros El Ermitaño, Jalisco, México. Revista Biología Tropical, 53: 229-238.

Hernández Vázquez, S., R. Rodríguez-Estrella, J. H. Vega-Rivera, F. Hernández-Vázquez, J. A. Rojo-Vázquez \& V. H. Galván- Piña. 2010. Estructura, dinámica y reproducción de las asociaciones de aves acuáticas de la costa de Jalisco, México. Pp 151-188. In: E. Godínez-Domínguez., C. Franco-Gordo., J.A. Rojo-Vázquez., F. Silva-Bátiz and G. González-Sansón (Eds.). Ecosistemas marinos de la costa Sur de Jalisco y Colima. Universidad de Guadalajara, México.

Howes, J. \& D. Bakewell. 1989. Shorebirds studies manual. Asian Wetland Bureau, Publication No. 55. Kuala Lumpur, Malaysia.

Mann, K. M. \& J. R. N. Lazier. 1991. Dynamics of marine ecosystem. Biological-physical interactions in the oceans. Blackwell Scientific Populations. Boston.

Mattiucci, S., M. Paoletti., J.Olivero-Verbel., R. Baldrini., B. Arroyo-Salgado., L. Garbin., G. Navone \& G. Nascetti. 2008. Contracaecum bioccai n. sp. from the brown pelican Pelecanus occi- 
dentalis (L.) in Colombia (Nematoda: Anisakidae): morphology, molecular evidence and its genetic relationship with congeners from fish-eating birds. Systematic Parasitology, 69: 101-121.

Miller, G. 2009. Confused pelicans may have lingered too long Up North. Science, 323: 449-449.

Parsons, T. R., Y. Maita. \& C. M. Lalli. 1984. A manual of chemical and biological methods for seawater analysis. Pergamon Press, Great Britain.

Rebón-Gallardo, F. 2000. Distribución, abundancia y conservación de la avifauna de las islas Marietas, Nayarit, México. Anales del Instituto de Biología, Serie Zoología, 71: 59-88.

Reeves, W. K., A. D. Loftis., F. Sanders., M. D. Spinks., W. Wills., A. M. Denison \& G. A. Dasch. 2006. Borrelia, Coxiella, and Rickettsia in Carios capensis (Acari: Argasidae) from a brown pelican (Pelecanus occidentalis) rookery in South Carolina, USA. Experimental Applied Acarology, 39: 321-329.

Rodgers, J. A. Jr. y S. T. Schwikert. 2002. Buffer-zone distance to protect foraging and loafing waterbirds from disturbance by personal watercraft and outboard-powered boats. Conservation Biology, 16: 216-224.

Rzedowski, J. 1978. Vegetación de México. Editorial Limusa, México, D. F.

Sánchez-Hernández, C. \& L. A. Pérez-Jiménez. 1972. Notas sobre la biología de la "Bubia de vientre blanco" (Sula leucogaster nesiotes; familia:Sulidae) en la Bahía Chamela, Jalisco, México. Revista de la Sociedad Mexicana de Historia Natural, Vol. 33. México.

Schreiber, R. W. 1980. The Brown Pelican: An endangered species? BioScience, 30: 742-747.

Schreiber, E. A. \& J. Burger. 2002. Biology of marine birds. Florida: CRC Press.

Shealer, D. A. 2002. Foraging behavior and food of seabirds. Pp 137-177. In: E.A. Schreiber and J. Burger (Eds.). Biology of marine birds. CRC PRESS, Washington, D.C.

Shields, M. A. 2002. Brown Pelican (Pelecanus occidentalis). In: A. Poole and F. Gill F (Eds.). The Birds of North America, No. 609: Philadelphia, Pennsylvania.

U.S. Fish and Wildlife Service. 2009. Endangered and Threatened Wildife and Plants; Removal of the Brown Pelican (Pelecanus occidentalis) From the Federal List of Endangered and Threatened Wildlife. Volume 74, No. 220. http://frwebgate.access.gpo.gov/cgi-bin/getdoc.cgi?dbname=2009 register\&docid $=$ fr17no09-14.

Wickliffe, J. K. \& J. W. Bickham. 1998. Flow cytometric analysis of hematocytes from Brown Pelicans (Pelecanus occidentalis) exposed to planar halogenated hydrocarbons and heavy metals. Bulletin of Environmental Contamination and Toxicology, 61: 239-246.

Work, T. M., B. Barr, A. M. Beale, L. Fritz., M. A. Quilliam \& J. L. C. Wright. 1993. Epidemiology of domoic acid poisoning in Brown Pelicans (Pelecanus occidentalis) and Brandt's Cormorants (Phalacrocorax penicilliatus) in California. Journal of Zoo and Wildlife Medicine, 24: 54-62.

Zamparo, Z., R. M. Overstreet \& D. R. B. Source. 2005. A new species of Petasiger (Digenea: Echinostomiformes: Echinostomatidae) in the Brown Pelican, Pelecanus occidentalis (Aves: Pelecaniformes: Pelecanidae), from the Area de Conservation Guanacaste, Costa Rica. The Journal of Parasitology, 91: 1465-1467.

Zar, J. H. 1974. Biostatistical Analysis. 2a edición. Prentice Hall, New York. 